\title{
Comparison of Graft Uptake and Postoperative Hearing Results between Butterfly-Cartilage and Temporalis-Fascia Myringoplasty
}

\author{
Amiya K Sah', Pabina Rayamajhi², Hari Bhattarai ${ }^{2}$ \\ ${ }^{1}$ Department of ENT- Head \& Neck Surgery, Janakpur Zonal Hospital, Janakpurdham, Nepal, ${ }^{2}$ Department of \\ ENT- Head \& Neck Surgery, Maharajgunj Medical Campus, Ganesh Man Singh Memorial Academy of ENT - Head \\ \& Neck Studies, Institute of Medicine, Maharajgunj, Kathmandu, Nepal
}

\section{Corresponding author:}

\section{Pabina Rayamajhi, MBBS, MS}

Ganesh Man Singh Memorial Academy of ENT- Head \& Neck Studies, Maharajgunj Medical Campus, Institute of Medicine, Kathmandu, Nepal

Email: pavina.rayamajhi@gmail.com

Submitted : Jan 2, 2020

Accepted : Feb 26, 2020

\begin{abstract}
Introduction

Myringoplasty is one of the commonest surgeries done in Otology for chronic otitis media- mucosal type (COM-mucosal type). The objective of this study was to compare the graft uptake and post-operative hearing status between butterfly-cartilage and temporalis-fascia myringoplasty.
\end{abstract}

\section{Methods}

Patients of age $\geq 15$ years with COM-mucosal type with dry, small to medium sized central perforation of pars tensa with clearly visible perforation margin were randomized into two groups of butterfly-cartilage myringoplasty and temporalis-fascia myringoplasty. Graft uptake and hearing results were assessed after six weeks and results were compared within and between the groups.

\section{Results}

Thirty-one patients in butterfly cartilage group and twenty-nine patients in temporalis fascia group were analysed. Graft uptake rate was $93.5 \%(29 / 31)$ in butterfly group and it was $86.2 \%(25 / 29)$ in temporalis fascia group with no statistically significant difference $(p=0.34)$ between the two groups. The mean pre and post-operative air bone gap in butterfly cartilage group were $20.65 \mathrm{~dB} \pm 8.35 \mathrm{~dB} ; 12.86 \mathrm{~dB} \pm 7.39 \mathrm{~dB}$ and that in the temporalis fascia group were $19.72 \pm 6.14 \mathrm{~dB}, 13.16 \mathrm{~dB} \pm 6.26 \mathrm{~dB}$ respectively. The difference of preoperative and postoperative hearing status, obtained within the two groups was statistically highly significant $(p<0.001)$. However, there was no statistically significant difference in hearing results between the two groups $(p=0.54)$.

\section{Conclusion}

The graft uptake rate and hearing results of butterfly-cartilage group and temporalis-fascia group were statistically significant within the group after myringoplasty but there was no statistically significant difference in between the two methods.

Keywords: Air bone gap, air conduction threshold, butterfly cartilage, myringoplasty 


\section{INTRODUCTION}

$\mathrm{M}$ yringoplasty for the closure of pars tensa was first performed by Berthold in 1878 and it was Wullstein and Zollner who popularized it through different types of tympanoplasty. ${ }^{1}$ The graft material has evolved over time from skin graft to grafts like vein, temporalis fascia and cartilage perichondrium composite grafts etc. $^{2}$

At present, temporalis fascia is the most frequently used graft material for myringoplasty. But, according to Beutner et al, the temporalis fascia too had few drawbacks which led to search for the better option of grafting. ${ }^{3}$ Jansen and Salen first used cartilage composite graft for tympanic membrane reconstruction in 1963. ${ }^{4}$ At the same time, Goodhill et al used cartilage perichondrium composite graft for the reconstruction. Since then various techniques have evolved for myringoplasty using cartilage such as cartilage palisade, ring graft, cartilage/perichondrium island graft, butterfly graft and shield technique. ${ }^{5}$

Among the various modifications in cartilage use, Eavey 1998 introduced cartilage-perichondrium butterfly tympanoplasty for closure of small to medium size perforations. This method used composite tragal perichondrium cartilage graft which is specially designed like butterfly to fit in the perforation without support in middle ear or external auditory canal.6,7 Inlay butterfly cartilage tympanoplasty (IBCT) has various practical advantages. This technique doesn't require the elevation of tympanomeatal flap, can be done in myringosclerotic tympanic membrane, no need of elevation of tympanomeatal flap and incision only in the tragus thus enhancing the aural comfort, tidiness, time saving and cost effective. This IBCT technique is expanding and is being used by many surgeons. However, this technique also has some limitations as it is difficult to perform in patients in whom the entire perforation margin cannot be viewed via the transcanal approach, inability to assess the ossicular chain, difficulty in large perforation with absence of rim of remnant tympanic membrane. According to Hod et $\mathrm{a}^{8}$ and Lin et $a^{9}{ }^{9}$, hearing level gain and air bone gap (ABG) closure in IBCT are as good as underlay temporalis fascia procedure.

There are various graft materials and techniques available to repair the central perforation and many studies have been done using different graft materials but very few comparative studies are found between butterfly cartilage and temporalis fascia. Thus, the aim of our study is to compare the feasibility of inlay butterfly cartilage without raising the tympanomeatal flap over temporalis fascia in small to medium sized perforation.

\section{METHODS}

This was a randomized-controlled, interventional study performed in patients who underwent myringoplasty from January 2014 to August 2015 in the Department of Ear Nose Throat (ENT) - Head \& Neck Surgery, Ganesh Man Singh Memorial Academy of ENT and Head \& Neck Studies, TU Teaching Hospital. Approval from the Institutional Review Board was taken for conducting the study.

The sample size was calculated using sample size calculator. The inclusion criteria for this study were all gender, age fifteen years and above, dry small to medium sized central perforations ${ }^{10}$ with wide external auditory canal and clearly visible margin of perforations. Large perforation, only hearing ear, wet ear and revision cases were excluded from the study. The patients with chronic otitis media (COM) mucosal type meeting the inclusion criteria with the pure tone audiogram done within one-week period were included for study. Randomization into two groups was done prior to surgery by lottery method into butterfly cartilage (group A) and temporalis fascia (group B). All surgeries were performed under local anesthesia by permeatal approach.

In group A (butterfly-cartilage) perforation margins were freshened, scraping of the undersurface done and the size of perforation measured by using piece of gelfoam. Tragal cartilage with perichondrium was harvested as graft material and cut in same shape as the perforation but with $1 \mathrm{~mm}$ larger margin. The graft was scored into the shape of butterfly by incision in center of cartilage and parallel to two layers of perichondrium. Gelfoam pieces were packed in the middle ear. The graft was then inserted in a transcanal fashion, microscopically, in such a way that groove grasps the anterior edge of perforation first and then placed with one wing of butterfly above and one below the margins of perforation.

In group B (temporal fascia), temporal fascia of the ipsilateral ear was harvested. The margin of the perforation was refreshed and the undersurface of remnant tympanic membrane made raw. Tympanomeatal flap was elevated by giving the incision in the bony external auditory canal about $5 \mathrm{~mm}$ lateral to annulus and it was carried from 6 o'clock position to 12 o'clock position. Middle ear and ossicular status were evaluated. Gelatin sponge (Gel foam) was kept in the middle ear and graft placed medial to the handle of malleus and remnant of tympanic membrane by underlay technique. Flap was repositioned back.

In both the groups, gel foam then bismuth-iodineparaffin pack was placed in the external auditory canal after graft placement for 7 days. The patients were followed up at regular interval till the seventh day for wound dressing, tuning fork test and any complications that might develop. The pack was 
Table 1. Demographic and operative data of patients

\begin{tabular}{lccc}
\hline & $\begin{array}{c}\text { Butterfly } \\
\text { cartilage } \\
\text { Group A } \\
(\mathbf{n = 3 1 )}\end{array}$ & $\begin{array}{c}\text { Temporalis } \\
\text { fascia } \\
\text { Group B } \\
(\mathbf{n}=29)\end{array}$ & $\begin{array}{c}p \text { - } \\
\text { value }\end{array}$ \\
\hline $\begin{array}{l}\text { Mean age } \\
\text { (years) }\end{array}$ & $29.19 \pm 14.20$ & $31.09 \pm 11.95$ & 0.70 \\
$\begin{array}{l}\text { Gender } \\
\text { Male }\end{array}$ & $17(54.82 \%)$ & $15(51.71 . \%)$ & 0.80 \\
$\begin{array}{l}\text { Female } \\
\text { Perforation }\end{array}$ & $14(45.26 \%)$ & $14(48.32 \%)$ & \\
$\begin{array}{l}\text { size } \\
\text { Small }\end{array}$ & $5(16.1 \%)$ & $1(3.4 \%)$ & 0.10 \\
Medium & $26(83.9 \%)$ & $28(96.6 \%)$ & \\
\hline
\end{tabular}

removed in the seventh day and patient kept on betamethasone and neomycin combination ear drops for another two weeks.

The patients were followed up for graft uptake and hearing assessment after 6 weeks. Graft uptake result was noted and pure tone audiometry (PTA) was performed. Total graft uptake was regarded as successful graft uptake. Residual perforation of any size was reported as failure. For hearing assessment; average of four frequency air bone gap and air conduction threshold was used to compare the pre and postoperative hearing results between the two groups. The air bone gap postoperative was measured as the gap between the preoperative bone conduction and postoperative air conduction threshold.

Statistical analysis was performed using SPSS 20 version. The average post-operative graft uptake between the two groups were analysed by Chi square test and hearing threshold change was compared using paired t test.

\section{RESULTS}

Total of 60 patients were randomized into two groups by lottery, with 31 patients in butterfly cartilage myringoplasty group (Group A) and 29 patients in temporalis fascia myringoplasty group (Group B).
Table 2. Comparison of postoperative graft uptake between two groups

\begin{tabular}{lccc}
\hline $\begin{array}{c}\text { Graft } \\
\text { outcome }\end{array}$ & $\begin{array}{c}\text { Butterfly } \\
\text { cartilage } \\
\text { Group A } \\
(\mathbf{n}=31)\end{array}$ & $\begin{array}{c}\text { Temporalis } \\
\text { fascia } \\
\text { Group B } \\
(\mathbf{n}=29)\end{array}$ & $p$-value \\
\hline Uptake & $29(93.5 \%)$ & $25(86.2 \%)$ & 0.34 \\
Failure & $2(6.5 \%)$ & $4(13.8 \%)$ & \\
\hline
\end{tabular}

The age of the patients enrolled ranged between 15 to 66 years with mean age of 29.35 years (SD 13.07 years). The most common age group was 15-30 years in both the groups. There was male predominance in both groups. The male to female ratio was 1.21 in group $A$ and 1.07 in group $B$. The maximum number of patients had medium sized perforation in both the groups. The above findings are tabulated as in Table 1. The demographic pattern compared between the groups showed no statistical difference.

Postoperatively, the total graft uptake in the butterfly cartilage (group A) was in $93.5 \%$ of the patients and $86.2 \%$ of the patients in the temporalis fascia (group B). Thus, it showed a slightly higher graft uptake rate in the cartilage group but the difference in the two group was not statistically significant with $p$-value of 0.34 as shown in Table 2 .

Both the air bone gap ( $A B$ gap) and air conduction threshold (AC threshold) showed significant improvement after surgery in both the butterfly cartilage group and temporalis fascia group $(p<0.001)$. However, pre-operative and postoperative hearing improvement between the two groups was not statistically significant. Summary of pre-operative and post-operative mean hearing between two groups are given in Table 3 .

\section{DISCUSSION}

This study was a randomized controlled study done in patients undergoing myringoplasty. There are limited number of comparative studies done in the past on butterfly cartilage and temporalis fascia myringoplasty and majority of studies were retrospective.

Table 3. Preoperative and postoperative mean hearing between two groups.

\begin{tabular}{lccc}
\hline & $\begin{array}{c}\text { Butterfly cartilage } \\
\text { Group } A(n=31)\end{array}$ & $\begin{array}{c}\text { Temporalis fascia } \\
\text { Group } B(n=29)\end{array}$ & $p$-value \\
\hline Pre-Op AC threshold & $36.17 \pm 11.94 \mathrm{~dB}$ & $37.00 \pm 10.30 \mathrm{~dB}$ & 0.46 \\
Post Op AC threshold & $27.93 \pm 10.43 \mathrm{~dB}$ & $29.32 \pm 8.31 \mathrm{~dB}$ & 0.67 \\
Pre-Op AB gap & $20.65 \pm 8.35 \mathrm{~dB}$ & $19.72 \pm 6.14 \mathrm{~dB}$ & 0.23 \\
Post Op AB gap & $12.86 \pm 7.39 \mathrm{~dB}$ & $13.16 \pm 6.26 \mathrm{~dB}$ & 0.59 \\
\hline
\end{tabular}


The follow up period of graft uptake in our study had mean of 3.17 months in group $A$ and 4.21 months in group $B$. The mean follow-up period in our study was similar to studies of Mauri et al ${ }^{11}$, Ozbek et al ${ }^{12}$ and Effat et $\mathrm{al}^{13}$.

The graft uptake rate of $93.5 \%$ in butterfly cartilage and $86.2 \%$ in fascia in our study is comparable with most of published series. Maheshwari et all4, 2015 in their prospective randomized study had achieved graft uptake rate of $96.9 \%$ in tragal cartilage composite group $(n=65)$ and $92.3 \%$ in temporalis fascia group $(n=65)$ with mean age of 26.4 years at the end of follow up period of 1 year. Our study was comparable to his study in terms of randomization, mean age distribution and results but differed in sample size, surgical approach, follow up period and results. They had larger sample size, exclusive postaural approach, single surgeon and longer duration of follow up. They also differed in surgical technique in terms of thinning the tragal cartilage $<0.5 \mathrm{~mm}$ thickness, shaped like island and placed by anterior tucking. In our study cartilage was kept in its full thickness and not thinned out but a groovelike butterfly shape was made and placed by inlay technique.

Wang et $\mathrm{al}^{15}, 2008$ had done retrospective study comparing inlay butterfly cartilage and temporalis fascia underlay myringoplasty in small to medium sized perforation of tympanic membrane. They achieved graft uptake rate of $82.1 \%$ in inlay butterfly cartilage group and $85 \%$ in temporalis fascia underlay group with no statistically significant difference $(p=0.79)$. Their study was different than ours in terms of lesser sample size, mean age distribution and longer follow up period and slightly lesser graft uptake rate.

A retrospective study done by Effat et al ${ }^{13}$ compared results of inlay butterfly cartilage with inlay temporalis fascia in both children and adults. They achieved graft uptake rate of $43 \%$ in inlay butterfly group and $83 \%$ in underlay temporalis fascia group over the mean follow up period of eight months. They had lower success rate of graft uptake in the both groups which was statistically significant. Better learning curve and shorter duration of follow up might be the reason for high success rate in fascia group than butterfly cartilage group.

Another randomized control trial done by Mauri et al ${ }^{11}$ compared inlay butterfly cartilage with underlay temporalis fascia myringoplasty in adults with medium sized tympanic membrane perforation. There were 34 patients in inlay butterfly cartilage group and 36 patients in underlay temporalis fascia group enrolled in the study. The graft uptake rate didn't differ between groups in one month with graft uptake rate of $88.2 \%$ in inlay butterfly cartilage group and $86.1 \%$ in underlay temporalis fascia group $(p=0.8)$. After a mean follow up period of 7.5 months the graft uptake rate was $85.3 \%$ inlay butterfly cartilage group and $83.3 \%$ in underlay temporalis fascia group. The study highlighted the importance of long term follow up in truly judging the graft uptake rate.

For hearing assessment, air conduction thresholds and air bone gap were taken. This is followed by most of the authors for objective hearing assessment. Several studies have used air-bone gap closure also in their study.

The hearing results obtained in our study was similar to the results of many other articles. Wang et al ${ }^{15}$ found $A B G$ closure of $6.3 \pm 2.5 \mathrm{~dB}$ in inlay butterfly group and $9.3 \pm 3.2 \mathrm{~dB}$ in underlay temporalis fascia group without statistically significant difference $(p=0.07)$ which was comparable to our study.

Study done by Couligner et $\mathrm{al}^{16}{ }^{16}$, found post-operative air bone gap closure at $2 \mathrm{KHz}, 14 \pm 10 \mathrm{~dB}$ in butterfly cartilage group and $13 \pm 6 \mathrm{~dB}$ in temporalis fascia group. There was no difference in hearing between the two groups. Mauri et al,11 found mean postoperative $A B G$ of $<20 \mathrm{~dB}$ in $94.1 \%$ patients in butterfly cartilage group and $97.2 \%$ patients in temporalis fascia group. There was no significant difference in hearing between two groups observed which was similar to our study.

Similarly, other studies done by Kim et al ${ }^{17}$, Eren et $\mathrm{al}^{18}$, Yu et $\mathrm{al}^{19}$, Apuhan et $\mathrm{al}^{20}$ and Shrestha et $\mathrm{al}^{21}$ also had reported significant hearing improvement observed after butterfly cartilage myringoplasty. But these studies showed the graft uptake of butterfly cartilage and lack the comparison with temporalis fascia.

Thus, majority of the studies in various literatures show the comparable hearing results between the butterfly cartilage group and temporalis fascia group. There had always been the uncertainty regarding cartilage to affect hearing status as it has a property of rigidity, mass and stiffness. But similar to our study and other literatures show comparable hearing to fascia grafting.

Our study has its limitation that it addresses the short-term graft uptake and audiologic results which might change with time as shown in few studies. There are evidences mentioned in literatures suggesting the improvement of postoperative hearing status in cartilage myringoplasty with time. Thus, a longer follow up period would be ideal to clear these doubts.

\section{CONCLUSION}

The graft uptake rate and hearing results of butterfly cartilage group are comparable to those of temporalis fascia group. Thus, butterfly cartilage myringoplasty can be used as an alternative option for the repair of small and medium size perforation of tympanic membrane which would save time and the need to raise the tympanomeatal flap. 


\section{CONFLICT OF INTEREST}

None declared.

\section{REFERENCES}

1. Wullstein HL. Functional operations in middle ear with split-thickness skin graft. Arch Otorhinolaryngol. 1952; 161:422-35.

2. Zollner F. The principles of plastic surgery of the sound-conducting apparatus. J Laryngol Otol. 1955; 69:657-9.

3. Beutner $\mathrm{D}$, Huttenbrink KB, Stumpf $\mathrm{R}$ et al. Cartilage Plate Tympanoplasty. Otology \& Neurotology. 2009; 31:105-110.

4. Yung M. Cartilage tympanoplasty: literature review. J Laryngol Otol. 2008; 122:663-72.

5. Neumann A, Kevenhoerster K, Gostian AO. LongTerm Results of Palisade Cartilage Tympanoplasty. Otology \& Neurotology. 2010; 31:936-939.

6. Anand TS, Kathuria G, Kumar S, Wadhawa B, Pradhan T. Butterfly inlay tympanoplasty: A study in Indian scenario. Indian journal of otolaryngology and Head and Neck surgery. 2002;54.

7. Eavey RD. Inlay tympanoplasty: cartilage butterfly technique. Laryngoscope. 1998;108: 657-61.

8. Hod R, Buda I, Hazan A, Negeris B. Inlay butterfly cartilage tympanoplasty. American journal of Otolaryngology - Head and Neck Medicine and Surgery. 2013; 34:41-43.

9. Lin YC, Wang HW, Weng HH, Lin YC. Predictors of Surgical and Hearing Long-term Results for Inlay Cartilage Tympanoplasty. Arch Otolaryngol Head Neck Surg. 2011; 137:215-219.

10. Singh GB, Sharma A, Singh N. Role of trans tympanic myringoplasty in modern otology. The journal of Otolaryngology. 2006; 35:408-12.

11. Mauri M, Neto JFL, Fuchs SC. Evaluation on inlay Butterfly Cartilage Tympanoplasty: A randomized clinical trial. The Laryngoscope. 2001.

12. Ozbek C, Ciftci O, Tuna EU et al. A Comparison of cartilage palisades and fascia in type I tympanoplasty in children: Anatomic and Functional Results. Otology \& Neurotology. 2008; 29:679-683.

13. Effat KG. Results of Inlay cartilage myringoplasty in terms of closure of central tympanic membrane perforations. The journal of Laryngology \& Otology. 2005; 119:611-613.

14. Maheshwari A, Panigrahi $R$, Mahajan $S$. Comparison of temporalis fascia with tragal cartilage - perichondrium (composite graft) as a grafting material in type I tympanoplasty. A prospective randomized study. International Journal of Scientific Study. 2015;3(1).

15. Wang $W H$, Lin YC. Minimally invasive inlay and underlay tympanoplasty. American journal of Otolaryngology.2008; 29(6):363-366.

16. Couloigner $V$, Baculard F, Bakkouri WE et al. Inlay butterfly cartilage tympanoplasty in children. Otology and neurotology. 2005; 26:247-51.

17. Kim SC, Park SH, Kim YC et al. The results of myringoplasty using cartilage butterfly technique (Inlay Tympanoplasty). Korean J OtolaryngolHead and Neck Surg.2001;44: (10):1038-1042.

18. Eren SB, Tugrul S, Ozucer B, Dogan R, Ozturan $O$. A randomized prospective trial of a novel device for measuring perforation size during inlay butterfly myringoplasty. American Journal of Otolaryngology -Head and Neck Medicine and Surgery. 2014;35:305-8.

19. Yu MS, Yoon TH, Bilateral same -day surgery for otitis media: Inlay butterfly cartilage myringoplasty. Otolaryngol Head and Neck Surg.2010;143(5):669-672.

20. Apuhan T, Kaya MO, Simsek T, Kazaz H. Butterfly cartilage graft myringoplasty. Abant Med J. 2012;1(1):8-12.

21. Shrestha BL, Amatya RC, Shrestha I, Pokhrel M. Comparison of Pre and Post- Operative Hearing Results in Patients Undergone Modified Inlay Butterfly Cartilage Perichondrium Myringoplasty. Journal of Rhinolaryngo-Otology. 2013; 1:82-86. 